\title{
Arbitrary-order economic production quantity model with and without deterioration: generalized point of view
}

\author{
Mostafijur Rahaman' ${ }^{1}$ Sankar Prasad Mondal ${ }^{2}$, Ali Akbar Shaikh ${ }^{3}$, Ali Ahmadian ${ }^{4}$, Norazak Senu ${ }^{4}$ and \\ Soheil Salahshour ${ }^{5}$
}

\author{
"Correspondence: \\ ahmadian.hosseini@gmail.com \\ ${ }^{4}$ Institute for Mathematical \\ Research, Universiti Putra Malaysia, \\ Serdang, Malaysia \\ Full list of author information is \\ available at the end of the article
}

\begin{abstract}
The key objective of this paper is to study and discuss the application of fractional calculus on an arbitrary-order inventory control problem. Using the concepts of fractional calculus followed by fractional derivative, we construct different possible models like generalized fractional-order economic production quantity (EPQ) model with the uniform demand and production rate and generalized fractional-order EPQ model with the uniform demand and production rate and deterioration. Also, we show that the classical EPQ model is the particular case of the corresponding generalized fractional EPQ model. This greatly facilitates the researcher a novel tactic to analyse the solution of the EPQ model in the presence of fractional index. Furthermore, this attempt also provides the solution obtained through the optimization techniques after using the real distinct poles rational approximation of the generalized Mittag-Leffler function.
\end{abstract}

Keywords: Fractional derivative; Differential equation of arbitrary-order; EPQ model; Laplace transformation; Real distinct poles rational approximation of the generalized Mittag-Leffler function; Geometric programming for optimization

\section{Introduction}

The journey of the concept of fractional calculus (FC) has started in the seventeenth century. Newton and Leibnitz are considered to be the first researchers among the mathematicians for working in this direction in mathematics. Through many decades the concepts regarding FC has been used to illustrate many real life problems relating to various fields of science and economy. FC explores integrals and derivatives of functions. Here the order of differentiation may be real or complex and hence in a particular case it may be of integer order. These days, global interest in FC has seemed to be exponential. Due to the different results of fractional derivatives, FC has attained much attention for modelling of the image processes, and various fields of mathematics, economics, physics and engineering [1-7].

A differential equation is understood to be a fractional differential equation (FDE) when differential operator is of a fractional order. FC simplifies the idea of a derivative of the

(c) The Author(s) 2020. This article is licensed under a Creative Commons Attribution 4.0 International License, which permits use, sharing, adaptation, distribution and reproduction in any medium or format, as long as you give appropriate credit to the original author(s) and the source, provide a link to the Creative Commons licence, and indicate if changes were made. The images or other third party material in this article are included in the article's Creative Commons licence, unless indicated otherwise in a credit line to the material. If material is not included in the article's Creative Commons licence and your intended use is not permitted by statutory regulation or exceeds the permitted use, you will need to obtain permission directly from the copyright holder. To view a copy of this licence, visit http://creativecommons.org/licenses/by/4.0/ 
integer order to a derivative operator of an arbitrary-order (may be real or complex). Both in the theoretic and useful outcomes had sharp out that FDEs extant outstanding outcomes in solving the different multipart problems that stand up in the field of engineering and science. It was established by several authors (see, e.g., [8-10]). It has been established that the fractional-order modelling is mostly beneficial to symbolize systems where computational effect on productions, this quality is the most important gain [11]. For the solutions of FDEs, it is complicated to acquire the analytical solutions owing their nonlocal stuff of the fractional derivative. Therefore, some systems were altered to obtain assessed solutions such as spectral methods [12], variational iteration methods [13], differential transform method [14] and Adomian decomposition method [15]. In recent years, researchers have had much attention for the field of FDEs. Recently, Hajipour et al. [16] discussed an accurate discretization of a variable-order fractional reaction-diffusion equation. The variable-order fractional description of compression deformation of amorphous glassy polymers was considered by Meng et al. [17]. The results for the nonlinear dynamical systems within the generalized fractional derivatives with Mittag-Leffler kernel were illustrated by Baleanu et al. [18]. For application purposes a new fractional analysis on the interaction of HIV with CD4+ T-cells was treated by Jajarmi and Baleanu [19]. A new aspect of the poor nutrition in the life cycle within the FC was investigated by Baleanu et al. [20]. Suboptimal control of fractional-order dynamic systems with delay argument was investigated by Jajarmi and Baleanu [21]. Recently, some interesting work regarding very realistic and physical problems was done as an application of the fractionalor arbitrary-order derivative method [22]. A fractional epidemiological model has been studied to describe computer viruses by Singh et al. [23]. Thereafter, Singh et al. [24] extended the Biswas-Milovic (BM) model which plays a vital role to describe the long distance optical communications. In addition, a rumor spreading dynamical model in a social network has been described by Singh. et al. [25]. A diabetes model and its complications with the Caputo-Frarizo fractional derivative have been investigated by Singh et al. [26]. Also, there is some work related to the application of FC on some production inventory problems which is interesting [27-31]. Also, a solution algorithm of fractional DrinfeldSokolov-Wilson equation in the numerical approach was given by Singh et al. [32].

\subsection{Research gap between our work and related published work with our contribution}

There are few papers [28-31] on the application of FC on EOQ models. On comparison, the same work on EPQ model is a little rare [27]. Moreover, the existing literature does not explain clearly the importance of the introduction of fractional concept on inventory theory. Besides that, there is no detailed explanation of the analytical or numerical optimization on the generalized EPQ model. The present study tries to overcome this deficiency.

Apart from these, in this paper we introduce the generalized EPQ model with deterioration which is totally novel. For numerical and analytical optimization, we use in a real distinct pole approximation the Mittag-Leffler function which is given by Iyiola, AsanteAsamani and Wade [33].

The contribution of the current study is:

(i) The modification of the woks by Das and Roy [27], especially in numerical result and motivation for using arbitrary order.

(ii) Arbitrary-order generalization of an EPQ model with and without deterioration when only the rate change of the inventory problem is arbitrary. 
(iii) Application of a real distinct pole rational approximation to optimize the inventory problem with Mittag-Leffler function. This was not used by earlier researchers of the fractional-order inventory model.

\subsection{The idea for taking the use of fractional calculus in inventory model}

Firstly, though the concept of the FC is a little abstract, one of its finest physical interpretations is that it has power to remember the previous effects of the input in order to determine the current output. Again, in the real world production system, the demand varies with the environment and circumstances. One of the important issues for the marketing system is the memory effect. Generally, the selling of the products depends on the quality of the product as well as the attitude or dealing policy of the supplier to the customers. Due to the good previous experience, a consumer gains interest for buying a product. On the contrary, for the bad impression on product or supplier, the consumer's demand gradually decreases. Thus, the memory effect is an important issue for the management system and intuitionally the introduction of the FC is justified.

Secondly, our main objective in this study is to view some common EPQ models in more generalized form. In this perspective, arbitrary-order calculus is an important tool. Usually, an inventory model is described by differential equations of integer order. But one thing to remember is that the FC is not the calculus of fractional order only. Here, the order may be real, complex or in particular integer.

So, if we use the notion of fractional derivative, integration, and differential equation, then we have the following facilities.

First of all, the EPQ model described by FDE is more realistic as it illustrates the idea of memory effect of the previous experience. Secondly, introducing the fractional (arbitrary) differential equation to describe the model, we can extend the theory of EPQ. And lastly, the classical inventory model with integer-order differential equation can be described as a particular case of the fractional one.

\subsection{Structure of the paper}

Furthermore, the remaining structure of the paper is organized as follows. After a detailed discussion and a general overview on FC in Sect. 2, the notations, units and their descriptions are elaborated in Sect. 3. The inventory problem of EPQ type is defined in Sect. 4. Details discussion of mathematical modelling for EPQ in different scenario is illustrated in Sect. 5. Theoretical or analytical results of corresponding optimization of the problems are shown in Sect. 6. The numerical results are shown in Sect. 7. The concluding remarks are made in Sect. 8.

\section{General overview on fractional calculus}

\subsection{Riemann-Liouville fractional derivative}

Let $f$ be a real valued continuous function. Then the left Riemann-Liouville derivative of fractional order $\alpha$ is defined to be

$$
{ }_{a} D_{x}^{\alpha}(f(x))=\frac{1}{\Gamma(m-\alpha)}\left(\frac{d}{d x}\right)^{m} \int_{a}^{x}(x-t)^{(m-\alpha-1)} f(t) d t,
$$

where $x>0$ and $m<\alpha<m+1$. 
And the right Riemann-Liouville derivative of fractional order $\alpha$ is defined to be

$$
{ }_{a} D_{x}^{\alpha}(f(x))=\frac{1}{\Gamma(m-\alpha)}\left(-\frac{d}{d x}\right)^{m} \int_{x}^{a}(x-t)^{(m-\alpha-1)} f(t) d t,
$$

where $x>0$ and $m<\alpha<m+1$.

Remark 2.1 The basic difference of the $\mathrm{R}-\mathrm{L}$ derivative from the ordinary calculus is that the $\mathrm{R}-\mathrm{L}$ derivative of constant term is not equal to zero.

Remark 2.2 For $m=1$ the left and right Riemann-Liouville derivative will be of the form ${ }_{a} D_{x}^{\alpha}(f(x))=\frac{1}{\Gamma(1-\alpha)} \frac{d}{d x} \int_{a}^{x}(x-t)^{-\alpha} f(t) d t$, where $x>0$ and ${ }_{a} D_{x}^{\alpha}(f(x))=\frac{1}{\Gamma(1-\alpha)}\left(-\frac{d}{d x}\right) \int_{x}^{a}(x-$ $t)^{-\alpha} f(t) d t$, where $x>0$.

\subsection{Riemann-Liouville fractional integral}

Let $f$ be a real valued continuous function. Then the Riemann-Liouville integral of fractional order $\alpha$ is defined to be

$$
{ }_{a} D_{x}^{-\alpha}(f(x))=\frac{1}{\Gamma(\alpha)} \int_{a}^{x}(x-t)^{(\alpha-1)} f(t) d t, \quad \text { where } x>0 \text { and } m<\alpha<m+1 .
$$

Remark 2.3 For $a=0$ above we have the definition of the Riemann integral and for $a=-\infty$ the same is the definition of the Liouville integral.

\subsection{Memory dependent derivative}

Using the kernel, the derivative of any function can be written as

$$
D(f(x))=\int_{a}^{x} K(x-t) f^{\prime}(t) d t
$$

\section{Remark 2.4}

(1) If we take $K(x-t)=\delta(x-t)$, it gives the memory-less derivative i.e. a derivative of integer order.

(2) If we consider $K(x-t)=\frac{(x-t)^{(m-\alpha)}}{\Gamma(m-\alpha)}$, then we get the expression

$$
D_{a}^{\alpha}(f(x))=\int_{a}^{x} K(x-t) f^{m}(t) d t
$$

Here $f^{m}$ denotes the common derivative of $m$ th order.

(3) The fractional derivative is not a local property. The total effects of the $\alpha$ th order derivative on the interval $[a, x]$ describe the variation of a system in which the instantaneous change rate depends on the past state; it is called the "memory effect".

(4) The memory strength is controlled by $\alpha$. As $\alpha \rightarrow 1$, the system becomes weaker in the sense of memory and for $\alpha=1$ it becomes totally memory-less. Lower value of $\alpha$ indicates a long memory of the system.

\subsection{Laplace transformation of fractional derivative}

The differential equation of arbitrary-order is the generalization of the differential equation. There are several ideas to find the solution of a fractional differential equation and 
the Laplace transformation is one of them. In this section of our study, we give a brief discussion of the Laplace transformation of a fractional derivative and its consequence.

Definition 2.1 The Laplace transform of a function $f(t)$ is given by

$$
F(s)=L\{f(t) ; s\}=\int_{0}^{\infty} e^{-s t} f(t) d t .
$$

Definition 2.2 The inverse Laplace transform of a function $F(s)$ is given by

$$
f(t)=L^{-1}(F(s))=\int_{-\infty}^{\infty} e^{s t} F(s) d s .
$$

Corollary 2.1 The Laplace transformation of the derivative of the integer order $n$ is given by

$$
L\left\{f^{n}(t) ; s\right\}=s^{n} F(s)-\sum_{k=0}^{n-1} s^{k} f^{(n-k-1)}(0)=s^{n} F(s)-\sum_{k=0}^{n-1} s^{(n-k-1)} f^{k}(0) .
$$

\subsection{Laplace transformation of the Riemann-Liouville derivative and integral}

The Laplace transform associated with the Riemann-Liouville derivative having fractional order $p>0$ is given by

$$
L\left\{{ }_{0} D_{t}^{p} f(t) ; s\right\}=s^{p} F(s)-\sum_{k=0}^{n-1} s^{k}\left[{ }_{0} D_{t}^{p-k-1} f(0)\right], \quad \text { for } n-1 \leq p<n .
$$

The Laplace transform associated with the Riemann-Liouville integral having the order $p$, where $p>0$ is

$$
L\left\{{ }_{0} D_{t}^{-p} f(t) ; s\right\}=s^{-p} F(s) .
$$

\section{Corollary 2.2}

(1) For $n=1$ the last expression takes the form

$$
L\left\{{ }_{0} D_{t}^{p} f(t) ; s\right\}=s^{p} F(s)-{ }_{0} D_{t}^{p-1} f(0), \quad \text { where } 0 \leq p<1 .
$$

(2) For $n=2$ the last expression takes the form

$$
L\left\{{ }_{0} D_{t}^{p} f(t) ; s\right\}=s^{p} F(s)-{ }_{0} D_{t}^{p-1} f(0)-s\left[{ }_{0} D_{t}^{p-2} f(0)\right], \quad \text { where } 1 \leq p<2
$$

(3) The m-times differentiated Mittag-Leffler function is given by

$$
E_{\alpha, \beta}^{(m)}(z)=\sum_{k=0}^{\infty} \frac{(k+m) !}{k !} \frac{z^{k}}{\Gamma(\alpha k+\alpha m+\beta)} .
$$

Then $L\left\{t^{\alpha m+\beta-1} E_{\alpha, \beta}^{(m)}\left(a t^{\alpha}\right) ; s\right\}=\frac{m ! s^{\alpha-\beta}}{\left(s^{\alpha}-a\right)^{m+1}}$. 


\subsection{Gamma function}

Let $z \in \mathbb{C}$, then the Gamma function is given by

$$
\Gamma(z)=\int_{0}^{\infty} e^{-t} t^{z-1} d t, \quad \text { for } \operatorname{Re}(z)>0 .
$$

By splitting this integral, at a point $x \geq 0$, we obtain two incomplete gamma functions;

$$
\gamma(z, x)=\int_{0}^{x} e^{-t} t^{z-1} d t
$$

and $\Gamma(z, x)=\int_{x}^{\infty} e^{-t} t^{z-1} d t$

\section{Remark 2.5}

(1) $\gamma(z, x)+\Gamma(z, x)=\Gamma(z)$, for all $x \geq 0$ and for all $\operatorname{Re}(z)>0$.

(2) If we consider $\Gamma(1, x)=e^{-x}$ then $\gamma(1, x)=1-e^{-x}$.

\subsection{Beta function}

Let $z, w \in \mathbb{C}$ then the Beta function is given by

$$
\mathrm{B}(z, w)=\int_{0}^{1} t^{z-1}(1-t)^{w-1} d t, \quad \text { for } \operatorname{Re}(z), \operatorname{Re}(w)>0 .
$$

\subsection{The Mittag-Leffler function}

The exponential function $e^{z}$ has a great importance in the study of the differential equation of integer order. This can also be written in a series form, which is given by

$$
e^{z}=\sum_{k=0}^{\infty} \frac{z^{k}}{\Gamma(k+1)}
$$

More generally, we can consider the expression

$$
E_{\alpha}(z)=\sum_{k=0}^{\infty} \frac{z^{k}}{\Gamma(\alpha k+1)} \text {, which is named the Mittag-Leffler function, }
$$

and the further generalization

$$
E_{\alpha, \beta}(z)=\sum_{k=0}^{\infty} \frac{z^{k}}{\Gamma(\alpha k+\beta)}, \quad \text { where } \alpha, \beta \in \mathbb{C} \text { and } \operatorname{Re}(\alpha)>0 ;
$$

this is also called the Mittag-Leffler function.

\section{Remark 2.6}

(1) For the special case of $\alpha=1$ and $\beta=1$, we have $E_{1,1}(z)=e^{z}$.

(2) The Mittag-Leffler function plays a major role $n$ the study of the functional calculus.

Corollary 2.3 Let $z \in \mathbb{C}, \alpha, \beta \in \mathbb{C}, \operatorname{Re}(\alpha)>0$ and $m \in \mathbb{N}$, then the $m$-times differentiated Mittag-Leffler function is given by

$$
E_{\alpha, \beta}^{(m)}(z)=\sum_{k=0}^{\infty} \frac{(k+m) !}{k !} \frac{z^{k}}{\Gamma(\alpha k+\alpha m+\beta)} .
$$




\subsection{A real distinct pole rational approximation of the generalized Mittag-Leffler function}

A real distinct pole rational function is given by

$$
R^{*}(z)=\frac{1+a z}{(1-b z)(1-c z)}, \quad \text { where } a, b, c \in \mathbb{R} \text { and } a \neq b
$$

A real distinct pole rational approximation of the generalized Mittag-Leffler function,

$$
E_{\alpha, \beta}(-z)=\sum_{k=0}^{\infty} \frac{(-z)^{k}}{\Gamma(\alpha k+\beta)}
$$

is given by

$$
E_{\alpha, \beta}(-z) \approx \frac{1-a z}{\Gamma(\beta)(1+b z)(1+c z)}
$$

where $a=\frac{\Gamma(\beta)}{\Gamma(\alpha+\beta)}-b-c, b=\frac{\Gamma(\alpha+\beta)}{\Gamma(2 \alpha+\beta)}-\frac{1}{4}, c=\frac{\Gamma(\beta) \Gamma(2 \alpha+\beta)}{\Gamma(\alpha+\beta)(5 \Gamma(2 \alpha+\beta)-4 \Gamma(\alpha+\beta))}$.

Special cases

(1) $E_{\alpha}(z)=E_{\alpha, 1}(z) \approx \frac{1+a z}{(1-b z)(1-c z)}$.

Here $a=\frac{1}{\alpha \Gamma(\alpha)}-b-c, b=\frac{\Gamma(\alpha)}{2 \Gamma(2 \alpha)}-\frac{1}{4}, c=\frac{\Gamma(2 \alpha)}{\alpha \Gamma(\alpha)(5 \Gamma(2 \alpha)-2 \Gamma(\alpha))}$.

(2) $e^{z}=E_{1,1}(z) \approx \frac{1+\frac{5}{12} z}{\left(1-\frac{1}{4} z\right)\left(1-\frac{1}{3} z\right)}$.

Remark 2.7 This is useful for solving scalar linear fractional differential equations.

\section{Different notations, units and their description for inventory model}

To describe our proposed problem, we use the following notations with certain units and description (see Tables 1 and 2).

Table 1 EPQ model without deterioration

\begin{tabular}{lll}
\hline Notations & Units & Descriptions \\
\hline$c_{1}$ & $\boldsymbol{Z} /$ unit & Holding cost per unit time \\
$c_{3}$ & $\boldsymbol{Z} /$ unit & Ordering cost per unit time \\
$K$ & Units & Production rate per cycle \\
$D$ & Units & Demand rate per cycle \\
$T$ & Year & Total time cycle \\
$t_{1}$ & Year & Production time \\
$q_{\max }$ & Units & Highest level of inventory \\
TAC & $\boldsymbol{Z} /$ Year & Total average cost \\
TAC $\alpha, \beta$ & $\boldsymbol{Z} /$ Year & Total generalized average cost \\
$\alpha$ & Constant & The order of integration \\
$\beta$ & Constant & The order of differention \\
Decision variable for integer-order model \\
$T$ & Year & Total time cycle \\
Decision variable for arbitrary-order model \\
$T$ & Year & Total time cycle \\
$t_{1}$ & Year & Production time \\
\hline
\end{tabular}


Table 2 EPQ model with deterioration

\begin{tabular}{lll}
\hline Notations & Units & Descriptions \\
\hline$c_{h}$ & $\boldsymbol{Z}$ /unit & Holding cost per unit time \\
$c_{0}$ & $\boldsymbol{Z}$ /unit & Ordering cost per unit time \\
$c_{p}$ & $\boldsymbol{Z}$ /unit & Production cost per unit time \\
$K$ & Units & Production rate per cycle \\
$D$ & Units & Demand rate per cycle \\
$T$ & Year & Total time cycle \\
$t_{1}$ & Year & Production time \\
$q_{\text {max }}$ & Units & Highest level of inventory \\
$\theta_{1}$ & Constant & Rate of deterioration in $\left[0, t_{1}\right]$ \\
$\theta_{2}$ & Constant & Rate of deterioration in $\left[t_{1}, T\right]$ \\
TAC & $\boldsymbol{Z} /$ Year & Total average cost \\
TAC $\alpha, \beta$ & $\boldsymbol{Z} /$ Year & Total generalized average cost \\
$\alpha$ & Constant & The order of integration \\
$\beta$ & Constant & The order of differention \\
$D_{\text {Decision variable for integer/arbitrary-order model }}$ & Total time cycle \\
$T$ & Year & Production time \\
$t_{1}$ & Year &
\end{tabular}

\section{Defining the problem for inventory planning of EPQ type}

\subsection{Integer-order EPQ model without deterioration}

The classical (integer-order) EPQ model is developed under the following assumptions:

(i) Demand is deterministic and uniform.

(ii) No shortage is allowed.

(iii) Lead time is zero.

(iv) Production rate is finite.

(v) Planning horizon is infinite.

\subsection{Arbitrary-order EPQ model with deterioration}

Along with all the above-mentioned assumptions, here, the additional assumption is that the EPQ model is memory sensitive i.e. the demand depends on the memory of the customer with the previous experience concerned with the behaviour of the shopkeeper or the quality of the product etc.

\subsection{Integer-order EPQ model with deterioration}

Along with all the assumptions, mentioned in Section 4.1, the additional assumption is that the products will be deteriorated with different rates in production time and nonproductive time.

\subsection{Arbitrary-order EPQ model with deterioration}

Along with all the assumptions, described in Section 4.3, the additional assumption is that the EPQ model is memory sensitive i.e. the demand depends on the memory of the customer with the previous experience concerned with the behaviour of the shopkeeper or the quality of the product.

\section{Details discussion of mathematical modelling for EPQ in different scenario}

\subsection{Classical (integer-order) EPQ model without deterioration (Model 1)}

There are two parts of this whole cycle during the first scheduling period $T$. The production starts at $t=0$ and throughout the time interval $\left[0, t_{1}\right]$, the inventory level is increased 
Figure 1 EPQ model without deterioration

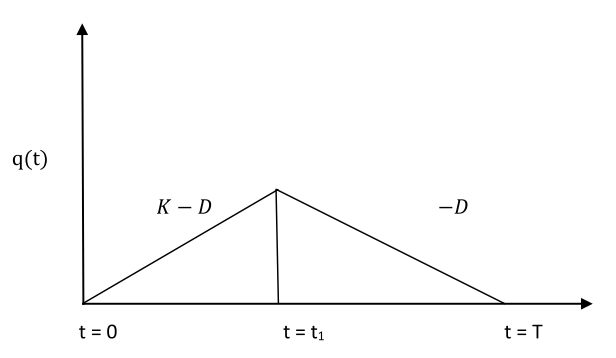

gradually at the rate $K-D$ due to production rate $K$ and to meet the demand the $D$. At the time $t=t_{1}$, the inventory reaches its highest level and here the production is stopped. Then during the time interval $\left[t_{1}, T\right]$, the inventory level gradually decreases as regards it being payable to happen up the customer's demands and at $t=T$ the inventory level reaches 0 , as no shortage is allowed (see Fig. 1).

If $q(t)$ represents the inventory level at any time, then the corresponding differential equation is given by

$$
\begin{aligned}
& \frac{d q(t)}{d t}=K-D, \quad \text { for } 0 \leq t \leq t_{1}, \\
& \frac{d q(t)}{d t}=-D, \quad \text { for } t_{1} \leq t \leq T .
\end{aligned}
$$

We have the boundary conditions $q(0)=q(T)=0$.

Now (5.1) along with the initial condition gives $q(t)=(K-D) t$, for $0 \leq t \leq t_{1}$.

Let $q\left(t_{1}\right)=q_{\max }$.

Now (5.2) along with the initial condition gives $q(t)=(T-t) D$, for $t_{1} \leq t \leq T$.

So, we have

$$
t_{1}=\frac{q_{\max }}{K-D}
$$

and

$$
T-t_{1}=\frac{q_{\max }}{D}
$$

So $T=t_{1}+\left(T-t_{1}\right)=q_{\max }\left(\frac{1}{K-D}+\frac{1}{D}\right)$, i.e.,

$$
q_{\max }=D\left(1-\frac{D}{K}\right) T
$$

\section{Some relevant costs}

(i) The holding cost is

$$
\begin{aligned}
\mathrm{HC} & =c_{1}\left[\int_{o}^{t_{1}} q(t) d t+\int_{t_{1}}^{T} q(t) d t\right] \\
& =c_{1}\left[(K-D) \frac{t_{1}^{2}}{2}+D \frac{\left(T-t_{1}\right)^{2}}{2}\right] \\
& =\frac{c_{1}}{2}\left(1-\frac{D}{K}\right) D T^{2}, \quad \text { using the last three equations. }
\end{aligned}
$$


(ii) The total cost is

$$
\mathrm{TC}=c_{1}+\frac{c_{1}}{2}\left(1-\frac{D}{K}\right) D T^{2} .
$$

(iii) The total average cost is

$$
\mathrm{TAC}=\frac{c_{3}}{T}+\frac{c_{1}}{2}\left(1-\frac{D}{K}\right) \mathrm{DT} .
$$

So, the classical EPQ model is

$$
\text { Minimize } \operatorname{TAC}(T)=\frac{c_{3}}{T}+\frac{c_{1}}{2}\left(1-\frac{D}{K}\right) \mathrm{DT} \text {. }
$$

Subject to $T>0$.

\subsection{Generalized (arbitrary-order) EPQ model without deterioration (Model 2)}

Here, we consider the memory effect with the same assumption and notations as for classical EPQ model. Then, the differential equation of fractional order $\alpha$, due to memory $(0 \leq \alpha \leq 1)$, corresponding to the generalized EPQ model, is

$$
\begin{aligned}
& \frac{d^{\alpha} q(t)}{d t^{\alpha}}=K-D, \quad \text { for } 0 \leq t \leq t_{1}, \\
& \frac{d^{\alpha} q(t)}{d t^{\alpha}}=-D, \quad \text { for } t_{1} \leq t \leq T,
\end{aligned}
$$

With the initial condition $q(0)=0$ and boundary condition $q(T)=0$.

Let

$$
q\left(t_{1}\right)=q_{\max }
$$

Taking the Laplace transformation of (5.10) we get

$$
s^{\alpha} \bar{q}(s)-s^{\alpha-0-1} q(0)=\frac{K-D}{s} .
$$

Or

$$
q(t)=L^{-1}(\bar{q}(s))=(K-D) \frac{t^{\alpha}}{\Gamma(\alpha+1)} .
$$

Then

$$
q\left(t_{1}\right)=q_{\max }=(K-D) \frac{t_{1}^{\alpha}}{\Gamma(\alpha+1)}
$$

Again, taking the Laplace transformation of (5.11) we have

$$
s^{\alpha} \bar{q}(s)-s^{\alpha-0-1} q\left(t_{1}\right)=\frac{-D}{s} .
$$


Or

$$
q(t)=L^{-1}(\bar{q}(s))=q_{\max }-D \frac{t^{\alpha}}{\Gamma(\alpha+1)} .
$$

Now, let $t_{2}=T-t_{1}$.

Again, $q(T)=0$ or

$$
q_{\max }=\frac{D T^{\alpha}}{\Gamma(\alpha+1)} .
$$

Hence, from (5.15) and (5.17) we have

$$
(K-D) \frac{t_{1}^{\alpha}}{\Gamma(\alpha+1)}=\frac{D T^{\alpha}}{\Gamma(\alpha+1)} .
$$

Then, for $0 \leq t \leq t_{1}$, we get

$$
q(t)=(K-D) \frac{t^{\alpha}}{\Gamma(\alpha+1)} .
$$

For $t_{1} \leq t \leq T$, we get

$$
q(t)=\frac{D}{\Gamma(\alpha+1)}\left[T^{\alpha}-t^{\alpha}\right]
$$

Now $q(t)$ is continuous at $t=t_{1}$.

So, $q_{\max }=q\left(t_{1}\right)$ or $(K-D) \frac{t_{1}^{\alpha}}{\Gamma(\alpha+1)}=\frac{D}{\Gamma(\alpha+1)}\left[T^{\alpha}-t_{1}^{\alpha}\right]$.

Or

$$
K t_{1}^{\alpha}=D T^{\alpha}
$$

Again, by (5.19) and (5.20) we get

$$
q_{\max }=\frac{D}{\Gamma(\alpha+1)}\left(1-\frac{D}{K}\right) T^{\alpha} .
$$

\section{Some relevant costs}

(i) The holding cost $\mathrm{HC}_{\alpha, \beta}(T)=c_{1} D^{-\beta} q(T)$.

Here

$$
\begin{aligned}
D^{-\beta} q(T)= & \frac{1}{\Gamma(\beta)} \int_{0}^{T}(T-x)^{\beta-1} q(x) d x \\
= & \frac{1}{\Gamma(\beta)}\left[\int_{0}^{t_{1}}(T-x)^{\beta-1} q(x) d x+\int_{t_{1}}^{T}(T-x)^{\beta-1} q(x) d x\right] \\
= & \frac{1}{\Gamma(\beta)}\left[\int_{0}^{t_{1}}(T-x)^{\beta-1}(K-D) \frac{x^{\alpha}}{\Gamma(\alpha+1)} d x\right. \\
& \left.+\int_{t_{1}}^{T}(T-x)^{\beta-1} \frac{D}{\Gamma(\alpha+1)}\left[T^{\alpha}-x^{\alpha}\right] d x\right] \\
= & \frac{1}{\Gamma(\beta)}\left(I_{1}+I_{2}\right) .
\end{aligned}
$$


Here

$$
I_{1}=\int_{0}^{t_{1}}(T-x)^{\beta-1}(K-D) \frac{x^{\alpha}}{\Gamma(\alpha+1)} d x
$$

and

$$
I_{2}=\int_{t_{1}}^{T}(T-x)^{\beta-1} \frac{D}{\Gamma(\alpha+1)}\left[T^{\alpha}-x^{\alpha}\right] d x
$$

So, the holding cost is

$$
\mathrm{HC}_{\alpha, \beta}(T)=\frac{c_{1}}{\Gamma(\beta)}\left(I_{1}+I_{2}\right)
$$

(ii) The total cost $=$ the holding cost + the set up cost

$$
=\frac{c_{1}}{\Gamma(\beta)}\left(I_{1}+I_{2}\right)+c_{3} .
$$

(iii) The total average cost, $\operatorname{TAC}_{\alpha, \beta}(T)=\frac{c_{1}}{\Gamma(\beta) T}\left(I_{1}+I_{2}\right)+\frac{c_{3}}{T}$.

Therefore, the model will be of the form

$$
\text { Minimize } \operatorname{TAC}_{\alpha, \beta}(T)=\frac{c_{1}}{\Gamma(\beta) T}\left(I_{1}+I_{2}\right)+\frac{c_{3}}{T}
$$

Such that $T>0$.

This is the generalized EPQ model.

\subsection{Classical (integer-order) EPQ model with deterioration (Model 3)}

During the first scheduling period $T$, there are two parts of this whole cycle. The production starts at $t=0$ and throughout the time interval $\left[0, t_{1}\right]$, the inventory level is increased at the rate $K-D$ due to the production rate $K$ and to meet the demand we have $D$. Also the deterioration affects the inventory level. In the time interval $\left[0, t_{1}\right]$, the deterioration rate is $\theta_{1}$. At the time $t=t_{1}$, the inventory reaches its highest level and the production is stopped. Then, throughout the time interval $\left[t_{1}, T\right]$, the inventory level gradually decreases as regards being payable to meet the customer's demands and for the deterioration at the rate $\theta_{2}$ and at $t=T$ the inventory level reaches 0 , as no shortage is allowed (see Fig. 2).

Figure 2 EPQ model with deterioration

$q(\mathrm{t})$

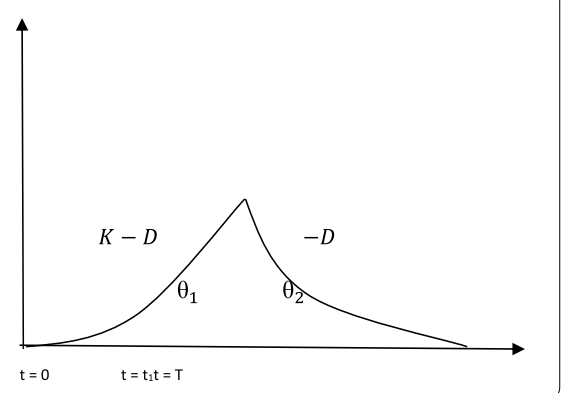


If $q(t)$ represents the inventory level at any time, then the corresponding differential equation is given by

$$
\begin{aligned}
& \frac{d q(t)}{d t}+\theta_{1} q(t)=K-D, \quad \text { for } 0 \leq t \leq t_{1}, \\
& \frac{d q(t)}{d t}+\theta_{2} q(t)=-D, \quad \text { for } t_{1} \leq t \leq T
\end{aligned}
$$

We have the boundary conditions

$$
q(0)=q(T)=0 .
$$

Also, let

$$
q_{\max }=q\left(t_{1}\right) .
$$

Solving Eqs. (5.28) and (5.29) and by using the boundary conditions we have

$$
\begin{aligned}
& q(t)=\frac{K-D}{\theta_{1}}\left(1-e^{-\theta_{1} t}\right), \quad \text { for } 0 \leq t \leq t_{1} . \\
& q(t)=\frac{D}{\theta_{2}}\left(e^{\theta_{2}(T-t)}-1\right), \quad \text { for } t_{1} \leq t \leq T .
\end{aligned}
$$

Then,

$$
q_{\max }=q\left(t_{1}\right)=\frac{K-D}{\theta_{1}}\left(1-e^{-\theta_{1} t_{1}}\right),
$$

Also, using the continuity condition we have

$$
\frac{K-D}{\theta_{1}}\left(1-e^{-\theta_{1} t_{1}}\right)=\frac{D}{\theta_{2}}\left(e^{\theta_{2}\left(T-t_{1}\right)}-1\right) .
$$

\section{Some relevant costs}

(i) The total holding cost is

$$
\begin{aligned}
\mathrm{HC} & =c_{h}\left[\int_{0}^{t_{1}} q(t) d t+\int_{t_{1}}^{T} q(t) d t\right] \\
& =c_{h}\left[\frac{K-D}{\theta_{1}} t_{1}+\frac{K-D}{\theta_{1}^{2}}\left(e^{-\theta_{1} t_{1}}-1\right)+\frac{D}{\theta_{2}^{2}}\left\{e^{\theta_{2}\left(T-t_{1}\right)}-\theta_{2}\left(T-t_{1}\right)-1\right\}\right] .
\end{aligned}
$$

(ii) The production cost,

$$
\mathrm{PC}=c_{p} \int_{0}^{t_{1}} K d t=c_{p} K t_{1} .
$$

(iii) Total cost of the system during the entire circle is given by

$$
X=c_{0}+\mathrm{HC}+\mathrm{PC} .
$$


(iv) Total average cost of the system during the entire circle is given by

$$
\mathrm{TAC}=\frac{X}{T}
$$

So, the problem can be written as

$$
\begin{gathered}
\text { Minimize TAC }=\frac{X}{T} . \\
\text { Subject to } T>0 .
\end{gathered}
$$

\subsection{Generalized (arbitrary-order) EPQ model with deterioration (Model 4)}

Here, we consider the memory effect with the same assumptions and notations as the integer-order EPQ model with deterioration. Then the differential equation of fractional order $\alpha$, due to memory $(0 \leq \alpha \leq 1)$, corresponding to the generalized EPQ model, is

$$
\begin{aligned}
& \frac{d^{\alpha} q(t)}{d t^{\alpha}}+\theta_{1} q(t)=K-D, \quad \text { for } 0 \leq t \leq t_{1}, \\
& \frac{d^{\alpha} q(t)}{d t^{\alpha}}+\theta_{2} q(t)=-D, \quad \text { for } t_{1} \leq t \leq T .
\end{aligned}
$$

We have the boundary conditions

$$
q(0)=q(T)=0
$$

Also, let

$$
q_{\max }=q\left(t_{1}\right) .
$$

Now, taking the Laplace transformation of (5.41) we have

$$
s^{\alpha} \bar{q}(s)-s^{\alpha-0-1} q(0)+\theta_{1} \bar{q}(s)=\frac{K-D}{s} .
$$

Or

$$
\bar{q}(s)=\frac{K-D}{\theta_{1}} \frac{\theta_{1}}{s\left(s^{\alpha}+\theta_{1}\right)} .
$$

Therefore, using the inverse Laplace transformation of (5.45) we have

$$
\begin{aligned}
q(t) & =L^{-1}(\bar{q}(s))=\frac{K-D}{\theta_{1}} L^{-1}\left(\frac{\theta_{1}}{s\left(s^{\alpha}+\theta_{1}\right)}\right) \\
& =\frac{K-D}{\theta_{1}}\left(1-E_{\alpha}\left(-\theta_{1} t^{\alpha}\right)\right), \quad \text { for } 0 \leq t \leq t_{1} .
\end{aligned}
$$

Here

$$
E_{\alpha}\left(-\theta_{1} t^{\alpha}\right)=\sum_{k=0}^{\infty} \frac{\left(-\theta_{1} t^{\alpha}\right)^{k}}{\Gamma(\alpha k+1)} .
$$


Now, taking the Laplace transformation of (5.42) we have

$$
s^{\alpha} \bar{q}(s)-s^{\alpha-0-1} q\left(t_{1}\right)+\theta_{2} \bar{q}(s)=\frac{-D}{s},
$$

Or

$$
\bar{q}(s)=\frac{s^{\alpha} q_{\max }}{s\left(s^{\alpha}+\theta_{2}\right)}-\frac{D}{\theta_{2}} \frac{\theta_{2}}{s\left(s^{\alpha}+\theta_{2}\right)} .
$$

Therefore, using the inverse Laplace transformation of (5.48) we have

$$
\begin{aligned}
q(t) & =L^{-1}(\bar{q}(s))=L^{-1}\left(\frac{s^{\alpha} q_{\max }}{s\left(s^{\alpha}+\theta_{2}\right)}\right)-\frac{D}{\theta_{2}} L^{-1}\left(\frac{\theta_{2}}{s\left(s^{\alpha}+\theta_{2}\right)}\right) \\
& =q_{\max } E_{\alpha}\left(-\theta_{2} t^{\alpha}\right)-\frac{D}{\theta_{2}}\left(1-E_{\alpha}\left(-\theta_{2} t^{\alpha}\right)\right), \quad \text { for } t_{1} \leq t \leq T
\end{aligned}
$$

Here

$$
E_{\alpha}\left(-\theta_{2} t^{\alpha}\right)=\sum_{k=0}^{\infty} \frac{\left(-\theta_{2} t^{\alpha}\right)^{k}}{\Gamma(\alpha k+1)}
$$

Now, let $t_{2}=T-t_{1}$.

$$
\text { Again, } q(T)=0 \text {. }
$$

So

$$
q_{\max }=\frac{D}{\theta_{2}} \frac{\left(1-E_{\alpha}\left(-\theta_{2} T^{\alpha}\right)\right)}{\left(E_{\alpha}\left(-\theta_{2} T^{\alpha}\right)\right)}=\frac{D}{\theta_{2}}\left\{\left(E_{\alpha}\left(-\theta_{2} T^{\alpha}\right)\right)^{-1}-1\right\} .
$$

Then (5.49) takes the form

$$
\begin{aligned}
q(t) & =\frac{D}{\theta_{2}}\left\{\left(E_{\alpha}\left(-\theta_{2} T^{\alpha}\right)\right)^{-1}-1\right\} E_{\alpha}\left(-\theta_{2} t^{\alpha}\right)-\frac{D}{\theta_{2}}\left(1-E_{\alpha}\left(-\theta_{2} t^{\alpha}\right)\right) \\
& =\frac{D}{\theta_{2}}\left\{E_{\alpha}\left(\theta_{2} T^{\alpha}\right) E_{\alpha}\left(-\theta_{2} t^{\alpha}\right)-1\right\} .
\end{aligned}
$$

Again, from (5.46) we have

$$
q_{\max }=q\left(t_{1}\right)=\frac{K-D}{\theta_{1}}\left(1-E_{\alpha}\left(-\theta_{1} t_{1}^{\alpha}\right)\right) .
$$

Now, from (5.51) and (5.52) we have

$$
\frac{K-D}{\theta_{1}}\left(1-E_{\alpha}\left(-\theta_{1} t_{1}^{\alpha}\right)\right)=\frac{D}{\theta_{2}}\left\{\left(E_{\alpha}\left(-\theta_{2} T^{\alpha}\right)\right)^{-1}-1\right\} .
$$

Then, for $0 \leq t \leq t_{1}$, we get

$$
q(t)=\frac{K-D}{\theta_{1}}\left(1-E_{\alpha}\left(-\theta_{1} t^{\alpha}\right)\right)
$$

and, for $t_{1} \leq t \leq T$, we get

$$
q(t)=\frac{D}{\theta_{2}}\left\{E_{\alpha}\left(\theta_{2} T^{\alpha}\right) E_{\alpha}\left(-\theta_{2} t^{\alpha}\right)-1\right\} .
$$

Now, $q(t)$ is continuous at $t=t_{1}$. 
So $q_{\max }=q\left(t_{1}\right)$ i.e.,

$$
\frac{K-D}{\theta_{1}}\left(1-E_{\alpha}\left(-\theta_{1} t_{1}^{\alpha}\right)\right)=\frac{D}{\theta_{2}}\left\{E_{\alpha}\left(\theta_{2} T^{\alpha}\right) E_{\alpha}\left(-\theta_{2} t_{1}^{\alpha}\right)-1\right\} .
$$

\section{Some relevant costs}

(i) The holding cost is

$$
\mathrm{HC}_{\alpha, \beta}(T)=c_{h} D^{-\beta} q(T)
$$

Here

$$
\begin{aligned}
D^{-\beta} q(T)= & \frac{1}{\Gamma(\beta)} \int_{0}^{T}(T-x)^{\beta-1} q(x) d x \\
= & \frac{1}{\Gamma(\beta)}\left[\int_{0}^{t_{1}}(T-x)^{\beta-1} q(x) d x+\int_{t_{1}}^{T}(T-x)^{\beta-1} q(x)\right] d x \\
= & \frac{1}{\Gamma(\beta)}\left[\int_{0}^{t_{1}}(T-x)^{\beta-1} \frac{K-D}{\theta_{1}}\left(1-E_{\alpha}\left(-\theta_{1} x^{\alpha}\right)\right) d x\right. \\
& \left.+\int_{t_{1}}^{T}(T-x)^{\beta-1} \frac{D}{\theta_{2}}\left\{E_{\alpha}\left(\theta_{2} T^{\alpha}\right) E_{\alpha}\left(-\theta_{2} x^{\alpha}\right)-1\right\} d x\right] \\
= & \frac{1}{\Gamma(\beta)}\left(I_{1}+I_{2}\right) .
\end{aligned}
$$

Here

$$
I_{1}=\int_{0}^{t_{1}}(T-x)^{\beta-1} \frac{K-D}{\theta_{1}}\left(1-E_{\alpha}\left(-\theta_{1} x^{\alpha}\right)\right) d x
$$

and

$$
I_{2}=\int_{t_{1}}^{T}(T-x)^{\beta-1} \frac{D}{\theta_{2}}\left\{E_{\alpha}\left(\theta_{2} T^{\alpha}\right) E_{\alpha}\left(-\theta_{2} x^{\alpha}\right)-1\right\} d x .
$$

(ii) The production cost is

$$
\begin{aligned}
\mathrm{PC} & =c_{p} D^{-\beta} K=c_{p} \frac{1}{\Gamma(\beta)} \int_{0}^{t_{1}}\left(t_{1}-x\right)^{\beta-1} K d x \\
& =c_{p} K \frac{1}{\Gamma(\beta)} \int_{0}^{t_{1}}\left(t_{1}-x\right)^{\beta-1} d x .
\end{aligned}
$$

(iii) The total cost of the system during the entire circle is given by

$$
\mathrm{TP}_{\alpha, \beta}(T)=c_{0}+\mathrm{HC}+\mathrm{PC} .
$$

(iv) The total average cost of the system during the entire circle is given by

$$
\operatorname{TAP}_{\alpha, \beta}(T)=\frac{\operatorname{TP}_{\alpha, \beta}(T)}{T} .
$$


So, the problem can be written as

$$
\begin{aligned}
& \text { Minimize } \operatorname{TAP}_{\alpha, \beta}(T)=\frac{\operatorname{TP}_{\alpha, \beta}(T)}{T} . \\
& \text { Subject to } T>0 .
\end{aligned}
$$

\section{Theoretical/analytical results for optimization of the problems}

\subsection{Model 1}

Solving a (5.9), we can show that $T^{*}=\sqrt{\frac{2 c_{3}}{C_{1} D\left(1-\frac{D}{K}\right)}}$ is the optimal cycle time at which the optimal average cost is $\operatorname{TAC}^{*}\left(T^{*}\right)=\sqrt{2 c_{1} c_{3} D\left(1-\frac{D}{K}\right)}$ and $q_{\max }^{*}=\sqrt{\frac{2 c_{3} D\left(1-\frac{D}{K}\right)}{C_{1}}}$.

\subsection{Model 2}

Case 6.1 $(\alpha=1$ and $\beta=1)$

(i) The holding cost

$$
\begin{aligned}
\mathrm{HC}_{1,1} & =\frac{c_{1}}{\Gamma(1)}\left[\int_{0}^{t_{1}}(T-x)^{1-1} q(x) d x+\right] \int_{t_{1}}^{T}(T-x)^{1-1} q(x) d x \\
& =\frac{c_{1}}{\Gamma(1)}\left[\int_{0}^{t_{1}}(K-D) \frac{x}{\Gamma(1+1)} d x+\int_{t_{1}}^{T} \frac{D}{\Gamma(1+1)}[T-x] d x\right] \\
& =c_{1}\left[(K-D) \frac{t_{1}^{2}}{2}+D \frac{\left(T-t_{1}\right)^{2}}{2}\right] \\
& =\frac{c_{1}}{2}\left(1-\frac{D}{K}\right) D T^{2}, \quad \text { using } K t_{1}=\mathrm{DT} .
\end{aligned}
$$

(ii) The total average cost $\mathrm{TAC}_{1,1}=\frac{c_{1}}{2}\left(1-\frac{D}{K}\right) \mathrm{DT}+\frac{c_{3}}{T}$.

So, the EPQ model is

$$
\text { Minimize } \operatorname{TAC}_{1,1}(T)=\frac{c_{1}}{2}\left(1-\frac{D}{K}\right) \mathrm{DT}+\frac{c_{3}}{T} .
$$

Such that $T>0$.

Remark 6.1 This is the classical EPQ model. It is seen that the classical EPQ model is a particular case of the general EPQ model.

Case 6.2 (When $\beta=1$ and $\alpha$ is any arbitrary number such that $0<\alpha \leq 1$ )

(i) The holding cost

$$
\begin{aligned}
\mathrm{HC}_{\alpha, 1}= & \frac{c_{1}}{\Gamma(1)}\left[\int_{0}^{t_{1}}(T-x)^{1-1} q(x) d x+\int_{t_{1}}^{T}(T-x)^{1-1} q(x) d x\right] \\
= & \frac{1}{\Gamma(1)}\left[\int_{0}^{t_{1}}(T-x)^{1-1}(K-D) \frac{x^{\alpha}}{\Gamma(\alpha+1)} d x\right. \\
& \left.+\int_{t_{1}}^{T}(T-x)^{1-1} \frac{D}{\Gamma(\alpha+1)}\left[T^{\alpha}-x^{\alpha}\right] d x\right] \\
= & c_{1} \frac{K-D}{\Gamma(\alpha+1)} \frac{t_{1}^{\alpha+1}}{(\alpha+1)}+c_{1} \frac{D}{\Gamma(\alpha+1)}\left[T^{\alpha+1}-\frac{T^{\alpha+1}}{\alpha+1}-T^{\alpha} t_{1}+\frac{t_{1}^{\alpha+1}}{\alpha+1}\right]
\end{aligned}
$$




$$
\begin{aligned}
= & \frac{\alpha c_{1}}{\Gamma(\alpha+2)} D\left[1-\left(\frac{D}{K}\right)^{\frac{1}{\alpha}}\right] T^{\alpha+1}, \\
& \text { using }(K-D) \frac{t_{1}^{\alpha}}{\Gamma(\alpha+1)}=\frac{D}{\Gamma(\alpha+1)}\left[T^{\alpha}-t_{1}^{\alpha}\right] \text { and } K t_{1}^{\alpha}=D T^{\alpha}
\end{aligned}
$$

(ii) The total average cost

$$
\begin{gathered}
\operatorname{TAC}_{\alpha, 1}=\frac{\alpha c_{1}}{\Gamma(\alpha+2)} D\left[1-\left(\frac{D}{K}\right)^{\frac{1}{\alpha}}\right] T^{\alpha}+\frac{c_{3}}{T}=c_{2} T^{\alpha}+\frac{c_{3}}{T}, \\
\text { where } \frac{\alpha c_{1}}{\Gamma(\alpha+2)} D\left[1-\left(\frac{D}{K}\right)^{\frac{1}{\alpha}}\right]=c_{2} .
\end{gathered}
$$

So, the EPQ model is

$\operatorname{Min} \operatorname{TAC}_{\alpha, 1}(T)=c_{2} T^{\alpha}+\frac{c_{3}}{T}$.

Such that $T>0$.

\section{Analytical solution using geometric programming method}

The corresponding dual problem is

$$
\text { Maximize } d(w)=\left(\frac{c_{2}}{w_{1}}\right)^{w_{1}}\left(\frac{c_{3}}{w_{2}}\right)^{w_{2}},
$$

subject to the normalized and orthogonal conditions

$$
\begin{aligned}
& w_{1}+w_{2}=1, \quad \alpha w_{1}-w_{2}=0, \\
& w_{1}, w_{2} \geq 0 .
\end{aligned}
$$

This gives $w_{1}=\frac{1}{\alpha+1}$ and $w_{2}=\frac{\alpha}{\alpha+1}$.

Also, the primal-dual relationship will be

$$
c_{2} T^{\alpha}=w_{1} d(w), \quad \frac{c_{3}}{T}=w_{2} d(w) .
$$

This gives $T=\left(\frac{c_{3}}{c_{2} \alpha}\right) \frac{1}{\alpha+1}$ and

$$
\text { Maximize } d(w)=c_{2}^{\frac{1}{\alpha+1}} c_{3}^{\frac{\alpha}{\alpha+1}} \alpha^{-\frac{\alpha}{\alpha+1}}(\alpha+1)
$$

So, finally the problem will be

$$
\text { Minimize } \operatorname{TAC}_{\alpha, 1}(T)=c_{2}^{\frac{1}{\alpha+1}} c_{3}^{\frac{\alpha}{\alpha+1}} \alpha^{-\frac{\alpha}{\alpha+1}}(\alpha+1)
$$

$$
\text { and } T=\left(\frac{c_{3}}{c_{2} \alpha}\right)^{\frac{1}{\alpha+1}}, q_{\max }=\frac{D}{\Gamma(\alpha+1)}\left(1-\frac{D}{K}\right) T^{\alpha}=\frac{D}{\Gamma(\alpha+1)}\left(1-\frac{D}{K}\right)\left(\frac{c_{3}}{c_{2} \alpha}\right)^{\frac{1}{\alpha+1}} \text {. }
$$


Case 6.3 (When $\alpha=1$ and $\beta$ is any arbitrary number such that $0<\beta \leq 1$ ) Therefore, the model will be of the form

$$
\operatorname{Min} \operatorname{TAC}_{1, \beta}(T)=\frac{c_{1}}{\Gamma(\beta) T}\left(I_{1}+I_{2}\right)+\frac{c_{3}}{T} .
$$

Such that $T>0$.

Here

$$
\begin{aligned}
I_{1} & =(K-D) \int_{0}^{t_{1}} x(T-x)^{\beta-1} d x=(K-D) \int_{0}^{t_{1}}\left[T(T-x)^{\beta-1}-(T-x)^{\beta}\right] d x \\
& =(K-D)\left[\frac{T^{(\beta+1)}-\left(T-t_{1}\right)^{\beta}\left(T+\beta t_{1}\right)}{\beta(\beta+1)}\right]
\end{aligned}
$$

and

$$
I_{2}=D \int_{t_{1}}^{T}(T-x)^{\beta} d x=-\frac{D\left(T-t_{1}\right)^{(\beta+1)}}{(\beta+1)} .
$$

Case 6.4 (For arbitrary values of $\alpha$ and $\beta$ the analytical illustration for the model is a little tough. For the sake of simplicity we take particular values of $\alpha$ and $\beta$. Here in our discussion we take $\alpha=0.5$ and $\beta=0.5$ ) Then,

$$
\begin{aligned}
I_{1} & =\frac{(K-D)}{\Gamma(1.5)} \int_{0}^{t_{1}}(T-x)^{-0.5} x^{0.5} d x=\frac{(K-D)}{\Gamma(1.5)}\left[T \sin ^{-1} \sqrt{\frac{t_{1}}{T}}-\sqrt{t_{1}\left(T-t_{1}\right)}\right] \\
I_{1} & =\frac{D}{\Gamma(1.5)} \int_{t_{1}}^{T}(T-x)^{-0.5}\left(T^{0.5}-x^{0.5}\right) d x \\
& =\frac{D}{\Gamma(1.5)}\left[2 \sqrt{T\left(T-t_{1}\right)}-\frac{\pi}{2} T+T \sin ^{-1} \sqrt{\frac{t_{1}}{T}}-\sqrt{t_{1}\left(T-t_{1}\right)}\right] .
\end{aligned}
$$

So the holding cost is $\mathrm{HC}_{0.5,0.5}(T)=\frac{c_{1}}{\Gamma(0.5)}\left(I_{1}+I_{2}\right)$.

Therefore the model will be of the form

$$
\text { Minimize } \operatorname{TAC}_{0.5,0.5}(T)=\frac{c_{1}}{\Gamma(0.5) T}\left(I_{1}+I_{2}\right)+\frac{c_{3}}{T} .
$$

Such that $T>0$.

\subsection{Model 3}

We think that there is no need to illustrate further theoretical results after describing in detail the part of mathematical modelling.

\subsection{Model 4}

Case 6.5 (When $\alpha=1$ and $\beta=1$ )

(i) The holding cost is $\mathrm{HC}_{1,1}(T)=c_{h} D^{-1} q(T)$. 
Here

$$
\begin{aligned}
D^{-1} q(t)= & \frac{1}{\Gamma(1)} \int_{0}^{T}(T-x)^{1-1} q(x) d x=\left[\int_{0}^{t_{1}} q(x) d x+\int_{t_{1}}^{T} q(x)\right] d x \\
= & {\left[\int_{0}^{t_{1}} \frac{K-D}{\theta_{1}}\left(1-E_{1}\left(-\theta_{1} x^{1}\right)\right) d x\right.} \\
& \left.+\int_{t_{1}}^{T} \frac{D}{\theta_{2}}\left(E_{1}\left(-\theta_{2} x^{1}\right)-E_{1}\left(-\theta_{2} T^{1}\right)\right) d x\right] \\
= & \left(I_{1}+I_{2}\right) .
\end{aligned}
$$

Here

$$
\begin{aligned}
I_{1} & =\int_{0}^{t_{1}} \frac{K-D}{\theta_{1}}\left(1-E_{1}\left(-\theta_{1} x^{1}\right)\right) d x=\int_{0}^{t_{1}} \frac{K-D}{\theta_{1}}\left(1-e^{-\theta_{1} x}\right) d x \\
& =\frac{K-D}{\theta_{1}} t_{1}+\frac{K-D}{\theta_{1}^{2}}\left(e^{-\theta_{1} t_{1}}-1\right)
\end{aligned}
$$

and

$$
\begin{aligned}
I_{2} & =\int_{t_{1}}^{T}(T-x)^{1-1} \frac{D}{\theta_{2}}\left\{E_{1}\left(\theta_{2} T^{1}\right) E_{1}\left(-\theta_{2} x^{1}\right)-1\right\} d x \\
& \left.=\int_{t_{1}}^{T} \frac{D}{\theta_{2}}\left(e^{\theta_{2}(T-x)}-1\right)\right) d x \\
& =\frac{D}{\theta_{2}^{2}}\left\{e^{\theta_{2}\left(T-t_{1}\right)}-\theta_{2}\left(T-t_{1}\right)-1\right\} .
\end{aligned}
$$

This shows that it is the holding cost for the classical EPQ model with deterioration.

(ii) The production cost is

$$
\begin{aligned}
\mathrm{PC}=c_{p} D^{-1} K & =c_{p} \frac{1}{\Gamma(1)} \int_{0}^{t_{1}}\left(t_{1}-x\right)^{1-1} K d x \\
& =c_{p} K t_{1} .
\end{aligned}
$$

This is the production cost of the classical EPQ model with deterioration.

(iii) The total cost of the system during the entire circle is given by

$$
\mathrm{TC}_{1,1}(T)=c_{0}+\mathrm{HC}+\mathrm{PC}
$$

(iv) The total average cost of the system during the entire circle is given by

$$
\operatorname{TAC}_{1,1}(T)=\frac{\operatorname{TP}_{1,1}(T)}{T}
$$

So the problem can be written as

Minimize $\mathrm{TAC}_{1,1}(T)=\frac{\mathrm{TP}_{1,1}(T)}{T}$

Subject to $T>0$. 
Remark 6.2 The above discussion shows that the classical EPQ model with deterioration is a particular case of the generalized fractional EPQ model with deterioration.

Case 6.6 (When $\alpha$ is any arbitrary number such that $0<\alpha \leq 1$ and $\beta=1$ ) Here, we use the real distinct pole approximation of the Mittag-Leffler function to avoid complexity of the function.

(i) The holding cost is

$$
\begin{aligned}
\mathrm{HC}_{\alpha, 1}(T) \\
=c_{h} \int_{0}^{T} q(x) d x \\
=c_{h}\left[\int_{0}^{t_{1}} q(x) d x+\int_{t_{1}}^{T} q(x)\right] d x \\
=c_{h}\left[\int_{0}^{t_{1}} \frac{K-D}{\theta_{1}}\left(1-E_{\alpha}\left(-\theta_{1} x^{\alpha}\right)\right) d x+\int_{t_{1}}^{T} \frac{D}{\theta_{2}}\left\{E_{\alpha}\left(\theta_{2} T^{\alpha}\right) E_{\alpha}\left(-\theta_{2} x^{\alpha}\right)-1\right\} d x\right] \\
=c_{h}\left[\int_{0}^{t_{1}} \frac{K-D}{\theta_{1}}\left(1-\frac{1-a_{1}\left(\theta_{1} x^{\alpha}\right)}{\left(1+a_{2}\left(\theta_{1} x^{\alpha}\right)\right)\left(1+a_{3}\left(\theta_{1} x^{\alpha}\right)\right)}\right) d x\right. \\
\left.\quad+\int_{t_{1}}^{T} \frac{D}{\theta_{2}}\left\{\frac{1+b_{1}\left(\theta_{2} T^{\alpha}\right)}{\left(1-b_{2}\left(\theta_{2} T^{\alpha}\right)\right)\left(1-b_{3}\left(\theta_{2} T^{\alpha}\right)\right)} \frac{1-c_{1}\left(\theta_{2} x^{\alpha}\right)}{\left(1+c_{2}\left(\theta_{2} x^{\alpha}\right)\right)\left(1+c_{3}\left(\theta_{2} x^{\alpha}\right)\right)}-1\right\} d x\right] .
\end{aligned}
$$

Here

$$
\begin{array}{lll}
a_{1}=\frac{1}{\alpha \Gamma(\alpha)}-a_{2}-a_{3}, & a_{2}=\frac{\Gamma(\alpha)}{2 \Gamma(2 \alpha)}-\frac{1}{4}, & a_{3}=\frac{\Gamma(2 \alpha)}{\alpha \Gamma(\alpha)(5 \Gamma(2 \alpha)-2 \Gamma(\alpha))} \\
b_{1}=\frac{1}{\alpha \Gamma(\alpha)}-b_{2}-b_{3}, & b_{2}=\frac{\Gamma(\alpha)}{2 \Gamma(2 \alpha)}-\frac{1}{4}, & b_{3}=\frac{\Gamma(2 \alpha)}{\alpha \Gamma(\alpha)(5 \Gamma(2 \alpha)-2 \Gamma(\alpha))}
\end{array}
$$

and

$$
c_{1}=\frac{1}{\alpha \Gamma(\alpha)}-c_{2}-c_{3}, \quad c_{2}=\frac{\Gamma(\alpha)}{2 \Gamma(2 \alpha)}-\frac{1}{4}, \quad c_{3}=\frac{\Gamma(2 \alpha)}{\alpha \Gamma(\alpha)(5 \Gamma(2 \alpha)-2 \Gamma(\alpha))} .
$$

For a particular value of $\alpha$, say $\alpha=0.5$ we have the following:

$$
\mathrm{HC}_{0.5,1}(T)=c_{h}\left(I_{1}+I_{2}\right)
$$

where

$$
\begin{aligned}
I_{1}= & \int_{0}^{t_{1}} \frac{K-D}{\theta_{1}}\left(1-\frac{1-a_{1}\left(\theta_{1} x^{\alpha}\right)}{\left(1+a_{2}\left(\theta_{1} x^{\alpha}\right)\right)\left(1+a_{3}\left(\theta_{1} x^{\alpha}\right)\right)}\right) d x \\
= & \frac{K-D}{\theta_{1}} \\
& \times\left\{t_{1}+2 \frac{\left(a_{1} a_{2} a_{3} \theta_{1}^{4}\left(a_{2}-a_{3}\right) \sqrt{t_{1}}-a_{2}^{2} \theta_{1}^{3}\left(a_{3}+a_{1}\right) \log \left(a_{3} \theta_{1} \sqrt{t_{1}}+1\right)+a_{3}^{2} \theta_{1}^{3}\left(a_{2}+a_{1}\right) \log \left(a_{2} \theta_{1} \sqrt{t_{1}}+1\right)\right)}{a_{2}^{2} a_{3}^{2} \theta_{1}^{5}\left(a_{2}-a_{3}\right)}\right\}
\end{aligned}
$$


and

$$
\begin{aligned}
I_{2} & =\int_{t_{1}}^{T} \frac{D}{\theta_{2}}\left\{\frac{1+b_{1}\left(\theta_{2} T^{\alpha}\right)}{\left(1-b_{2}\left(\theta_{2} T^{\alpha}\right)\right)\left(1-b_{3}\left(\theta_{2} T^{\alpha}\right)\right)} \frac{1-c_{1}\left(\theta_{2} x^{\alpha}\right)}{\left(1+c_{2}\left(\theta_{2} x^{\alpha}\right)\right)\left(1+c_{3}\left(\theta_{2} x^{\alpha}\right)\right)}-1\right\} d x \\
& =\frac{D}{\theta_{2}}\left[\left\{\frac{1+b_{1}\left(\theta_{2} \sqrt{T}\right)}{\left(1-b_{2}\left(\theta_{2} \sqrt{T}\right)\right)\left(1-b_{3}\left(\theta_{2} \sqrt{T}\right)\right)}\right\}\left(\mathrm{J}_{3}-\mathrm{J}_{4}\right)-T+t_{1}\right] .
\end{aligned}
$$

Here

$$
\begin{aligned}
& \mathrm{J}_{3}=-2 \frac{\left(c_{1} c_{2} c_{3} \theta_{1}^{4}\left(c_{2}-c_{3}\right) \sqrt{T}-c_{2}^{2} \theta_{1}^{3}\left(c_{3}+c_{1}\right) \log \left(c_{3} \theta_{1} \sqrt{T}+1\right)+c_{3}^{2} \theta_{1}^{3}\left(c_{2}+c_{1}\right) \log \left(c_{2} \theta_{1} \sqrt{T}+1\right)\right)}{c_{2}^{2} c_{3}^{2} \theta_{1}^{5}\left(c_{2}-c_{3}\right)}, \\
& \mathrm{J}_{4}=-2 \frac{\left(c_{1} c_{2} c_{3} \theta_{1}^{4}\left(c_{2}-c_{3}\right) \sqrt{t_{1}}-c_{2}^{2} \theta_{1}^{3}\left(c_{3}+c_{1}\right) \log \left(c_{3} \theta_{1} \sqrt{t_{1}}+1\right)+c_{3}^{2} \theta_{1}^{3}\left(c_{2}+c_{1}\right) \log \left(c_{2} \theta_{1} \sqrt{t_{1}}+1\right)\right)}{c_{2}^{2} c_{3}^{2} \theta_{1}^{5}\left(c_{2}-c_{3}\right)} .
\end{aligned}
$$

(ii) The production cost is $\mathrm{PC}_{0.5,1}=c_{p} \int_{0}^{t_{1}} K d x=c_{p} K t_{1}$.

So the problem can be written as

Minimize $\operatorname{TAP}_{\alpha, 1}(T)=\frac{c_{0}+\mathrm{HC}+\mathrm{PC}}{T}$.

Subject to $T>0$.

Case 6.7 (When $\alpha=1$ and $\beta$ is arbitrary number such that $0<\beta \leq 1$ )

(i) The holding cost is $\mathrm{HC}_{1, \beta}(T)=c_{h} D^{-\beta} q(T)=\frac{c_{h}}{\Gamma(\beta)}\left(I_{1}+I_{2}\right)$.

Here

$$
\begin{aligned}
I_{1} & =\int_{0}^{t_{1}} \frac{K-D}{\theta_{1}}(T-x)^{\beta-1}\left(1-e^{-\theta_{1} x}\right) d x \\
& =\frac{K-D}{\theta_{1}}\left[\frac{T^{\beta}-\left(T-t_{1}\right)^{\beta}}{\beta}-e^{-\theta_{1} T}\left(-\theta_{1}\right)^{-\beta}\left\{\Gamma\left(\beta, \theta_{1}\left(t_{1}-T\right)\right)-\Gamma\left(\beta,-\theta_{1} T\right)\right\}\right]
\end{aligned}
$$

and

$$
\begin{aligned}
I_{2} & =\int_{t_{1}}^{T} \frac{D}{\theta_{2}}(T-x)^{\beta-1}\left(e^{\theta_{2}(T-x)}-1\right) d x \\
& =\frac{D}{\theta_{2}}\left[\left(-\theta_{2}\right)^{-\beta}\left\{\Gamma(\beta, 0)-\Gamma\left(\beta, \theta_{2}\left(t_{1}-T\right)\right)\right\}-\frac{\left(T-t_{1}\right)^{\beta}}{\beta}\right] .
\end{aligned}
$$

(ii) The production cost is $\mathrm{PC}_{1, \beta}(T)=c_{p} D^{-1} K=c_{p} \frac{1}{\Gamma(\beta)} \int_{0}^{t_{1}}\left(t_{1}-x\right)^{\beta-1} K d x$.

(iii) The total cost of the system during the entire circle is given by

$\mathrm{TC}_{1, \beta}(T)=c_{0}+\mathrm{HC}+\mathrm{PC}$.

So, the problem can be written as

Minimize $\operatorname{TAC}_{1, \beta}(T)=\frac{\operatorname{TP}_{1, \beta}(T)}{T}$.

Subject to $T>0$. 
Case 6.8 For arbitrary values of $\alpha$ and $\beta$ the problem becomes more complicated. But for particular values of $\alpha$ and $\beta$ the problem can be solved numerically with approach described in Case 6.6.

\section{Numerical results and sensitivity analysis}

Some numerical examples have been taken and sensitivity analysis on the examples has been done to justify the theoretical aspects.

\subsection{Model 1}

For numerical illustration, we take the following numerical values:

$$
c_{1}=4, \quad c_{3}=30, \quad K=2500, \quad D=1200 .
$$

Then the minimum total average cost is $\mathrm{TAC}^{*}=386.9884$ which is obtained for the optimal time cycle $T^{*}=96.74709$. Also then the maximum inventory level $q_{\max }^{*}=96.74709$.

In Table 3, the sensitivity of the optimality with respect to the values of the parameters is shown.

From Table 3, we can make the following observations:

(i) As the values of $c_{1}$ increase, $T^{*}$ gradually decreases and $\mathrm{TAC}^{*}$ gradually increases. This indicates that as the holding cost increases the total schedule time has to decrease to minimize the total average cost.

Table 3 Sensitivity table for case Model 1

\begin{tabular}{lllll}
\hline Parameters & Change in values & $T^{*}$ & TAC & $q_{a m x}^{*}$ \\
\hline$c_{1}$ & 3.6 & 0.1634301 & 367.1294 & 101.9804 \\
& 3.8 & 0.1590712 & 377.1896 & 99.26042 \\
& 3.9 & 0.1570186 & 382.1204 & 97.97959 \\
4 & 0.1550434 & 386.9884 & 96.74709 \\
4.1 & 0.1531410 & 391.7959 & 95.55997 \\
& 4.2 & 0.1513069 & 396.5451 & 94.41550 \\
& 4.3 & 0.1495372 & 401.2381 & 93.31118 \\
$C_{3}$ & 27 & 0.1470871 & 367.1294 & 91.78235 \\
& 28 & 0.1497862 & 373.8663 & 93.46657 \\
& 29 & 0.1524375 & 380.4839 & 95.12098 \\
& 30 & 0.1550434 & 386.9884 & 96.74709 \\
& 31 & 0.1576063 & 393.3853 & 98.34633 \\
& 32 & 0.1601282 & 399.6799 & 99.91997 \\
& 33 & 0.1626109 & 405.8768 & 101.4692 \\
& 2470 & 0.1559199 & 384.8129 & 96.20323 \\
& 2480 & 0.1556237 & 385.5453 & 96.38632 \\
& 2490 & 0.1553316 & $386 / 2704$ & 96.56760 \\
& 2500 & 0.1550434 & 386.9884 & 96.74709 \\
& 2510 & 0.1547591 & 387.6993 & 96.92483 \\
& 2520 & 0.1544786 & 388.4033 & 97.10083 \\
& 2530 & 0.1542018 & 389.1005 & 97.27513 \\
& & 0.1552376 & 386.5043 & 96.62608 \\
& 1170 & 0.1551628 & 386.6906 & 96.67264 \\
& 1180 & 0.1550981 & 386.8519 & 96.71298 \\
& 1190 & 0.1550434 & 386.9884 & 96.74709 \\
& 1200 & 0.1549987 & 387.1000 & 96.77500 \\
& 1210 & 0.1549640 & 387.1868 & 96.79669 \\
& 1220 & 0.1549392 & 387.2488 & 96.81219 \\
\hline 1230 & & & \\
& & & &
\end{tabular}


(ii) As the values of $c_{3}$ increase, both $T^{*}$ and TAC* gradually increase. This fact can be interpreted as follows: if the ordering cost increases then to minimize the total average cost the inventory procedure needs to run a long time.

(iii) As the value of $K$ increases, $T^{*}$ gradually decreases and $\mathrm{TAC}^{*}$ gradually increases. That means that, for a high production rate, the total schedule time must be shorter and the total average cost will be minimized.

(iv) As the values of $D$ increases, $T^{*}$ gradually decreases and TAC* gradually increases. That means that to meet a large demand the total time must be shorter and then obviously the total average cost will be minimized.

\subsection{Model 2}

We consider the same numerical value as taken for Model 1 . Then, for this arbitrary model we have the following cases:

Case 6.1: When $\alpha=1$ and $\beta=1$

In the theoretical discussion we have shown that for this case Model 2 reduces to Model 1. The same thing happens for the numerical example also, i.e., if we take the same numerical example as Model 1 with $\alpha=1$ and $\beta=1$ then we can illustrate Model 2 using Model 1.

Case 6.2: When $\alpha>0$ is arbitrary and $\beta=1$

Here we discuss the same numerical example as Model 1, i.e., $c_{1}=4, c_{3}=30, K=2500$, $D=1200$.

In Table 4, the sensitivity of the optimality with respect to the different values of the memory index $\alpha$ is shown.

From Table 4, we can make the observation that there is a critical value of the memory parameter, say $=0.3$, for which the minimized total average cost becomes maximum (480.7231) and then the value of TAC* decreases above and below the table. The lower values of $\alpha$ indicates a large memory. So, another interpretation from the table is that for large memory the system needs more time to minimize the total average cost. That means that to reach the same minimum cost like the memory-less inventory system, the dealer has to change the policy of dealing with customers.

In Table 5 , the sensitivity of the optimality with respect to the value of the parameters is shown for the fixed values of the memory parameters $\alpha=0.5$ and $\beta=1$.

Table 4 Results for $\alpha>0$ with the fixed value $\beta=1$ of Case 6.2

\begin{tabular}{lllclll}
\hline$\alpha$ & $\Gamma(\alpha+2)$ & $\Gamma(\alpha+1)$ & $\frac{\alpha c_{1}}{\Gamma(\alpha+2)} D\left[1-\left(\frac{D}{K}\right) \frac{1}{\alpha}\right]=c_{2}$ & $T^{*}$ & TAC $^{*}$ & $q_{\max }^{*}$ \\
\hline 0.1 & 1.04648585469 & 0.9135076987 & 458.3802 & 0.6801931 & 319.2005 & 631.1130 \\
0.2 & 1.10180249088 & 0.9181687424 & 879.0985 & 0.2358365 & 446.3459 & 509.0779 \\
0.3 & 1.16671190520 & 0.8974706963 & 1127.364 & 0.1551386 & 480.7231 & 397.5395 \\
0.4 & 1.24216934450 & 0.8872638175 & 1298.952 & 0.1304171 & 476.9366 & 311.3620 \\
0.5 & 1.32934038818 & 0.8862269255 & 1389.441 & 0.1230857 & 460.6260 & 247.0264 \\
0.6 & 1.42962455886 & 0.8935153493 & 1421.718 & 0.1234093 & 441.9322 & 199.0175 \\
0.7 & 1.54468584585 & 0.9086387329 & 1412.893 & 0.1279405 & 414.5195 & 162.8167 \\
0.8 & 1.67649078776 & 0.9313837710 & 1375.371 & 0.1351746 & 409.5117 & 135.1363 \\
0.9 & 1.82735508062 & 0.9617658319 & 1318.186 & 0.1443518 & 397.0645 & 113.6567 \\
1 & 2.00000000000 & 1.0000000000 & 1248.000 & 0.1550434 & 386.9884 & 96.74709 \\
\hline
\end{tabular}


Table 5 Sensitivity table for Case 6.2 for fix $\alpha=0.5$ and $\beta=1$

\begin{tabular}{|c|c|c|c|c|}
\hline Parameters & Change in values & $T^{*}$ & TAC* & $q_{a m x}^{*}$ \\
\hline \multirow[t]{7}{*}{$c_{1}$} & 3.6 & 0.1320421 & 429.3816 & 255.8562 \\
\hline & 3.8 & 0.1273674 & 445.1409 & 251.2863 \\
\hline & 3.9 & 0.1251808 & 452.9165 & 249.1199 \\
\hline & 4 & 0.1230857 & 460.6260 & 247.0264 \\
\hline & 4.1 & 0.1210760 & 468.2714 & 245.0015 \\
\hline & 4.2 & 0.1191465 & 475.8550 & 243.0414 \\
\hline & 4.3 & 0.1172920 & 483.3786 & 241.1426 \\
\hline \multirow[t]{7}{*}{$c_{3}$} & 27 & 0.1147367 & 444.7295 & 238.5014 \\
\hline & 28 & 0.1175525 & 450.1535 & 241.4102 \\
\hline & 29 & 0.1203350 & 455.4500 & 244.2506 \\
\hline & 30 & 0.1230857 & 460.6260 & 247.0264 \\
\hline & 31 & 0.1258059 & 465.6882 & 249.7412 \\
\hline & 32 & 0.1284971 & 470.6127 & 252.3982 \\
\hline & 33 & 0.1311603 & 475.4950 & 255.0004 \\
\hline \multirow[t]{7}{*}{ K } & 2470 & 0.1236897 & 458.3764 & 244.8555 \\
\hline & 2480 & 0.1234851 & 459.1360 & 245.5850 \\
\hline & 2490 & 0.1232838 & 459.8858 & 246.3086 \\
\hline & 2500 & 0.1230857 & 460.6260 & 247.0264 \\
\hline & 2510 & 0.1228907 & 461.3568 & 247.7384 \\
\hline & 2520 & 0.1226988 & 462.0783 & 248.4448 \\
\hline & 2530 & 0.1225099 & 462.7902 & 249.1455 \\
\hline \multirow[t]{7}{*}{ D } & 1170 & 0.1239622 & 457.3688 & 247.2847 \\
\hline & 1180 & 0.1236581 & 458.4937 & 247.2192 \\
\hline & 1190 & 0.1233659 & 459.5795 & 247.1331 \\
\hline & 1200 & 0.1230857 & 460.6260 & 247.0264 \\
\hline & 1210 & 0.1220171 & 461.6330 & 246.8992 \\
\hline & 1220 & 0.1225603 & 462.6004 & 246.7515 \\
\hline & 1230 & 0.1223150 & 463.5280 & 246.5834 \\
\hline
\end{tabular}

Table 6 Result for Case 6.3

\begin{tabular}{lllll}
\hline$\beta$ & $T^{*}$ & $t_{1}^{*}$ & TAC $^{*}$ & $q_{\max }^{*}$ \\
\hline 0.1 & 0.9578852 & 0.4597849 & 344.5089 & 597.7204 \\
0.2 & 0.3246652 & 0.1558393 & 554.4172 & 202.5911 \\
0.3 & 0.2031685 & 0.9752090 & 639.8628 & 126.1772 \\
0.4 & 0.1614390 & 0.07749071 & 650.4005 & 100.7379 \\
0.5 & 0.1446220 & 0.06941856 & 622.3119 & 90.24313 \\
0.6 & 0.1386208 & 0.06653798 & 577.1140 & 86.49937 \\
0.7 & 0.1383842 & 0.06642440 & 526.4847 & 86.35172 \\
0.8 & 0.1417004 & 0.06801618 & 476.3573 & 88.4203 \\
0.9 & 0.1474590 & 0.07078033 & 429.4978 & 92.0143 \\
1.0 & 0.1550434 & 0.07442084 & 386.9884 & 96.74709 \\
\hline
\end{tabular}

The observations in Table 5 and the interpretations regarding it are the same as that of Table 3.

Case 6.3: When $\alpha=1$ and $\beta$ is arbitrary

Here we fix the value of $\beta=0.5$ and take the same example as Case 6.2. Then the minimum value of the total average cost is $\mathrm{TAC}^{*}=622.3119$, which is given for the optimal time cycle $T^{*}=0.1446220$. Also the maximum inventory will be $q_{\max }^{*}=90.24413$, which is obtained at $t_{1}^{*}=0.0694185$.

In Table 6, the sensitivity of the optimality with respect to the different values of the memory index $\beta$ is shown. 
From Table 6, we can make the observation that there is a critical value of the memory parameter, say 0.4 , for which the minimized total average cost becomes maximum (650.4005) and then the value of $\mathrm{TAC}^{*}$ decreases above and below the table. The lower values of $\beta$ indicate a large memory. So, another interpretation from the table is that for large memory the system needs more time to minimize the total average cost. That means that to reach the same minimum cost like the memory-less inventory system, the dealer has to change the policy of dealing with customers.

In Table 7, the sensitivity of the optimality with respect to the value of the parameters is shown for the fixed values of the memory parameters $\alpha=1$ and $\beta=0.5$.

The observations in Table 7 and the interpretations regarding it are the same as that of Table 3.

\section{Case 6.4: When $\alpha, \beta$ are arbitrary}

In our discussion we take $\alpha=0.5, \beta=0.5$ and $c_{1}=4, c_{3}=40, K=250, D=120$.

Then, the minimum value of the total average cost, $\mathrm{TAC}^{*}=146.3850$, which is given for the optimal time interval, $T^{*}=1.010695$ and $t_{1}^{*}=0.2562614$ and $q_{\max }^{*}=70.78638$.

In Table 8, the sensitivity of the optimality with respect to the value of the parameters is shown.

Table 7 Sensitivity table for Case 6.3 for fixed $\alpha=1$ and $\beta=0.5$

\begin{tabular}{lllllc}
\hline Parameters & Change in values & $T^{*}$ & $t_{1}^{*}$ & TAC $^{*}$ & $q_{\max }^{*}$ \\
\hline$c_{1}$ & 3.9 & 0.1470837 & 0.0760019 & 611.89 & 91.78025 \\
& 4 & 0.1446220 & 0.06941856 & 622.3119 & 90.24413 \\
& 4.1 & 0.1422608 & 0.06828517 & 632.3411 & 88.77972 \\
$c_{3}$ & 25 & 0.1280698 & 0.06147352 & 585.6180 & 79.91558 \\
& 30 & 0.1446220 & 0.06941856 & 622.3119 & 90.24413 \\
& 35 & 0.1602749 & 0.0769319 & 655.1243 & 100.0115 \\
$K$ & 2490 & 0.1449054 & 0.06983392 & 621.0949 & 90.08576 \\
& 2500 & 0.1446220 & 0.06941856 & 622.3119 & 90.24413 \\
& 2510 & 0.1443425 & 0.06900838 & 623.5168 & 90.40098 \\
& 1190 & 0.1448451 & 0.06894628 & 621.3533 & 90.31963 \\
& 1200 & 0.1446220 & 0.06941856 & 622.3119 & 90.24413 \\
& 1210 & 0.1444108 & 0.06989481 & 623.2223 & 90.16430 \\
\hline
\end{tabular}

Table 8 Sensitivity table for Case 6.4 for fixed $\alpha=0.5$ and $\beta=0.5$

\begin{tabular}{lllcl}
\hline Parameters & Change in values & $T^{*}$ & TAC* & $q_{\max }^{*}$ \\
\hline$C_{1}$ & 3.9 & 1.010935 & 143.7147 & 70.79477 \\
& 4 & 1.010695 & 146.3850 & 70.78638 \\
& 4.2 & 1.010246 & 151.7250 & 70.77065 \\
$C_{3}$ & 38 & 1.010223 & 144.4057 & 70.76986 \\
& 40 & 1.010695 & 146.3850 & 70.78638 \\
& 42 & 1.011161 & 148.3634 & 70.80270 \\
$K$ & 247 (non-feasible) & 0.02102814 & 2033.175 & 10.09586 \\
& 250 & 1.010695 & 146.3850 & 70.78638 \\
& 253 & 1.010959 & 146.9759 & 71.57052 \\
$D$ & 119 & 1.010960 & 145.9075 & 70.74572 \\
& 120 & 1.010695 & 146.3850 & 70.78638 \\
& 123 & 1.009944 & 147.7492 & 70.85533 \\
\hline
\end{tabular}


The observations in Table 8 and the interpretations regarding it are the same as that of Table 3 .

\subsection{Model 3}

We set an example given as follows:

$$
\begin{aligned}
& c_{h}=50, \quad c_{0}=500, \quad c_{p}=36, \quad K=25,000, \\
& D=15,000, \quad \theta_{1}=0.35, \quad \theta_{2}=0.005 .
\end{aligned}
$$

Then the minimum cost TAC $*$ is $561,866.9$, which is given for the optimal value of $T^{*}=$ 0.1454915 and $t_{1}^{*}=0.08783444$. Also, then the maximum inventory level will be $q_{\max }^{*}=$ 81,183 .

In Table 9, the sensitivity of the optimality with respect to the value of the parameters is shown.

From Table 9, we can make the following observations.

(i) When the value of $c_{h}$ increases then $T^{*}$ and $\mathrm{TAC}^{*}$ remain the same. So, we can say the optimality is stable with respect to the change of the holding cost.

(ii) When the value of $c_{0}$ increases then both $T^{*}$ and TAC* gradually increase. This fact can be interpreted as follows: if the ordering cost increases then to minimize the total average cost the inventory procedure needs to run a long time.

(iii) When the value of $c_{p}$ increases $\mathrm{TAC}^{*}$ gradually increases. This fact can be interpreted as follows: if the production cost increases, the total average cost increases.

Table 9 Sensitivity table for Model 3

\begin{tabular}{llllll}
\hline Parameters & Change in values & $T^{*}$ & $t_{1}^{*}$ & $T^{*}$ & $q_{\max }^{*}$ \\
\hline$c_{h}$ & 48 & 0.1454915 & 0.08783444 & $561,866.9$ & 81,183 \\
& 50 & 0.1454915 & 0.08783444 & $561,866.9$ & 81,183 \\
& 52 & 0.1454915 & 0.08783444 & $561,866.9$ & 81,183 \\
$c_{p}$ & 34 & 0.1417628 & 0.09164477 & $516,582.9$ & $81,162.12$ \\
& 36 & 0.1454915 & 0.08783444 & $561,866.9$ & 81,183 \\
& 40 & 0.1399389 & 0.0844624 & $607,139.6$ & $81,201.39$ \\
$c_{0}$ & 490 & 0.1440319 & 0.08694787 & $561,797.8$ & $81,187.85$ \\
& 500 & 0.1454915 & 0.08783444 & $561,866.9$ & 81,183 \\
& 510 & 0.1469366 & 0.0887122 & $561,935.3$ & $81,178.20$ \\
$K$ & 24,950 & 0.1455730 & 0.08805949 & $561,863.1$ & $80,771.88$ \\
& 25,000 & 0.1454915 & 0.08783444 & $561,866.9$ & 81,183 \\
& 25,050 & 0.1454113 & 0.08761109 & $561,870.6$ & $81,594.15$ \\
0 & 14,950 & 0.1456006 & 0.0876086 & $560,011.5$ & $81,596.95$ \\
& 15,000 & 0.1454915 & 0.08783444 & $561,866.9$ & 81,183 \\
& 15,050 & 0.1453860 & 0.0806192 & $563,722.1$ & $80,769.23$ \\
& 0.345 & 0.1465341 & 0.08845998 & $561,818.1$ & $83,556.77$ \\
$\theta_{1}$ & 0.35 & 0.1454915 & 0.08783444 & $561,866.9$ & 81,183 \\
& 0.355 & 0.1444709 & 0.08722204 & $561,915.3$ & $78,908.90$ \\
& 0.0045 & 0.1455589 & 0.08787488 & $561,863.7$ & $81,182.80$ \\
$\theta_{2}$ & 0.005 & 0.1454915 & 0.08783444 & $561,866.9$ & 81,183 \\
& 0.0055 & 0.1454242 & 0.08779406 & $561,870.1$ & $81,183.21$ \\
& & & & &
\end{tabular}


(iv) If the value of $K$ increases then $T^{*}$ gradually decreases and TAC* gradually increases. That means that for high production rate the total time must be shorter and the total average cost will be minimized.

(v) When the value of $D$ increases then $T^{*}$ gradually decreases and TAC* gradually increases. That means that to meet a large demand the total time must be shorter and then obviously the total average cost will be minimized.

(vi) When the values of $\theta_{1}, \theta_{2}$ increase then $T^{*}$ gradually decreases and TAC ${ }^{*}$ gradually increases. That means that as the total cost gradually increases due to deterioration, for minimum cost the procedure has to run for shorter time.

\subsection{Model 4}

Case 6.5: When $\alpha=1$ and $\beta=1$

In this case Model 4 is reduces to the model 3. So if we take the same numerical data as model-3 the numerical discussion will be done for this case.

Case 6.6: When $\alpha>0$ is arbitrary and $\beta=1$

For example if we take $\alpha=0.5$ and set an example given by

$$
\begin{aligned}
& c_{h}=50, \quad c_{0}=500, \quad c_{p}=36, \quad K=2500, \\
& D=1500, \quad \theta_{1}=0.5, \quad \theta_{2}=0.005,
\end{aligned}
$$

then the minimum cost is $\mathrm{TAC}_{0.5,1}^{*}=568575.9$, which is given for the optimal value of $T^{*}=9.978575 .9$ and $t_{1}^{*}=0.0004$.

In Table 10, the sensitivity of the optimality with respect to the value of the parameters is shown.

Table 10 Sensitivity table for Case 6.6 for fixed $\alpha=0.5$ and $\beta=1$

\begin{tabular}{llclc}
\hline Parameters & Change in values & $T^{*}$ & $t_{1}^{*}$ & TAC $C_{0.5,1}^{*}$ \\
\hline$c_{h}$ & 40 & 9.974794 & 0.0004 & $454,871.5$ \\
& 50 & 9.974794 & 0.0004 & $568,575.9$ \\
& 60 & 9.974795 & 0.0004 & $682,280.2$ \\
$c_{p}$ & 9.974794 & 0.0004 & $568,575.3$ \\
& 30 & 9.974794 & 0.0004 & $568,575.9$ \\
& 36 & 9.974794 & 0.0004 & $568,576.3$ \\
$c_{0}$ & 40 & 9.974795 & 0.0004 & $568,570.8$ \\
& 450 & 9.974794 & 0.0004 & $568,575.9$ \\
& 500 & 9.974795 & 0.0004 & $568,580.8$ \\
$K$ & 550 & 9.974795 & 0.000416666 & $511,600.2$ \\
& 2400 & 9.974794 & 0.0004 & $568,575.9$ \\
& 2500 & 9.974795 & 0.0003846154 & $625,570.2$ \\
0 & 2600 & 9.974795 & 0.0004 & $625,427.6$ \\
& 1400 & 9.974794 & 0.0004 & $568,575.9$ \\
& 1500 & 9.974795 & 0.0004 & $511,724.0$ \\
$\theta_{1}$ & 1600 & 77.14196 & 0.0004 & $171,729.7$ \\
& 0.4 & 9.974794 & 0.0004 & $568,575.9$ \\
& 0.5 & 27.81227 & 0.0004 & $99,140.86$ \\
$\theta_{2}$ & 0.6 & 9.912312 & 0.0004 & $572,159.9$ \\
& 0.004 & 9.974794 & 0.0004 & $568,575.9$ \\
& 0.005 & 10.03836 & 0.0004 & $564,975.5$ \\
\hline & 0.006 & & &
\end{tabular}


The observations in Table 10 and the interpretations regarding it are the same as that of Table 9.

\section{Conclusion}

In this paper, we realize that classical economic production quantity model (EPQ) may be generalized as a fractional-order EPQ model with and without deterioration. It is being perceived that holding costs and total average costs for non-fractional cases are the particular cases of generalized holding costs and generalized total average costs. That means that the classical EPQ model may be seen as a particular case of the generalized EPQ model. We have also seen that the generalized EPQ model (fractional EPQ) is not so easy to optimize analytically by any ordinary optimization method. It needs some different solution procedure. For that, we use the real distinct poles rational approximation method of the generalized Mittag-Leffler function and after simplifying we get the result of the optimization problem. In the future, we shall be looking for the analytical as well as numerical optimization method for fractional EPQ models. Hence the FC may be exploited to grow any other classical EPQ and EPQ model with different verity. Moreover, we conclude that the fractional-order inventory model mechanism has been successfully implied for a business which has been a new result. More work with practical info needs to be carried out for future features.

Funding

This research was financially supported by Ministry of Education, Malaysia under FRGS grant (Grant No.: 01-01-18-2031FR) and Universiti Putra Malaysia (UPM), Malaysia.

Availability of data and materials

All date generated or analysed during this study are included in this article.

Competing interests

The authors declare that they have no competing interests.

Authors' contributions

Each of the authors contributed equally to each part of this work. All authors read and approved the final manuscript.

\section{Author details}

${ }^{1}$ Department of Mathematics, Indian Institute of Engineering Science and Technology, Shibpur, Howrah, India.

${ }^{2}$ Department of Applied Science, Maulana Abul Kalam Azad University of Technology, West Bengal, Haringhata, India.

${ }^{3}$ Department of Mathematics, University of Burdwan, Burdwan, India. ${ }^{4}$ Institute for Mathematical Research, Universiti

Putra Malaysia, Serdang, Malaysia. ${ }^{5}$ Faculty of Engineering and Natural Sciences, Bahcesehir University, Istanbul, Turkey.

\section{Publisher's Note}

Springer Nature remains neutral with regard to jurisdictional claims in published maps and institutional affiliations.

Received: 31 January 2019 Accepted: 11 December 2019 Published online: 08 January 2020

\section{References}

1. Baleanu, D., Diethelm, K., Scalas, E., Trujillo, J.J.: Fractional Calculus: Models and Numerical Methods. World Scientific, Singapore (2012)

2. Gorenflo, R., Mainardi, F.: Fractional calculus. In: Fractals and Fractional Calculus in Continuum Mechanics, pp. 223-276 (1997)

3. Agila, A., Baleanu, D., Eid, R., Iranfoglu, B.: Applications of the extended fractional Euler-Lagrange equations model to freely oscillating dynamical systems. Rom. J. Phys. 61, 350-359 (2016)

4. Podlubny, I.: Fractional Differential Equations. Academic Press, San Diego (1999)

5. Agrawal, O.P., Tenreiro-Machado, J.A., Sabatier, I.: Fractional Derivatives and Their Applications. Nonlinear Dyn. 38 (2004)

6. Baleanu, D., Gven, Z.B., Tenreiro Machado, J.A.: New Trends in Nanotechnology and Fractional Calculus Applications. Springer, New York (2010)

7. Machado, J.A., Mata, M.E.: Pseudo phase plane and fractional calculus modeling of western global economic downturn. Commun. Nonlinear Sci. Numer. Simul. 22, 396-406 (2015) 
8. Kilbas, A.A., Srivastava, H.M., Trujillo, J.J.: Theory and Applications of Fractional Differential Equations. Elsevier, Amsterdam (2006)

9. Miller, K.S., Ross, B.: An Introduction to the Fractional Calculus and Differential Equations. Wiley, New York (1993)

10. Magin, R.L.: Fractional Calculus in Bioengineering. Begell House Publisher, Inc., Danbury (2006)

11. Mainardi, F., Pagnini, G., Gorenflo, R.: Some aspects of fractional diffusion equations of single and distributed order. Appl. Math. Comput. 187, 295-305 (2007)

12. Bhrawy, A.H., Tharwat, M.M., Yildirim, A.: A new formula for fractional integrals of Chebyshev polynomials: application for solving multi-term fractional differential equations. Appl. Math. Model. 37, 4245-4252 (2013)

13. Abbasbandy, S.: An approximation solution of a nonlinear equation with Riemann-Liouville's fractional derivatives by He's variational iteration method. J. Comput. Appl. Math. 207, 53-58 (2007)

14. Arikoglu, A., Ozkol, I.: Solution of fractional integro-differential equations by using fractional differential transform method. Chaos Solitons Fractals 40, 521-529 (2009)

15. Duan, J.S., Chaolu, T., Rach, R., Lu, L.: The Adomian decomposition method with convergence acceleration techniques for nonlinear fractional differential equations. Comput. Math. Appl. 66, 728-736 (2013)

16. Hajipour, M., Jajarmi, A., Baleanu, D., Sun, H.G.: On an accurate discretization of a variable-order fractional reaction-diffusion equation. Commun. Nonlinear Sci. Numer. Simul. 69, 119-133 (2019)

17. Meng, R., Yin, D., Drapaca, C.S.: Variable-order fractional description of compression deformation of amorphous glassy polymers. Comput. Mech. 64, 163-171 (2019)

18. Baleanu, D., Jajarmi, A., Hajipour, M.: On the nonlinear dynamical systems within the generalized fractional derivatives with Mittag-Leffler kernel. Nonlinear Dyn. 94(1), 397-414 (2018)

19. Jajarmi, A., Baleanu, D.: A new fractional analysis on the interaction of HIV with CD4+ T-cells. Chaos Solitons Fractals $113,221-229(2018)$

20. Baleanu, D., Jajarmi, A., Bonyah, E., Hajipour, M.: New aspects of the poor nutrition in the life cycle within the fractional calculus. Adv. Differ. Equ. 2018(1), 230 (2018)

21. Jajarmi, A., Baleanu, D.: Suboptimal control of fractional-order dynamic systems with delay argument. J. Vib. Control 24(12), 2430-2446 (2018)

22. Singh, J., Secer, A., Swroop, R., Kumar, D.: A reliable analytical approach for a fractional model of advection-dispersion equation. Nonlinear Eng. (2018). https://doi.org/10.1515/nleng-2018-0027

23. Singh, J., Kumar, D., Hammouch, Z., Atangana, A.: A fractional epidemiological model for computer viruses pertaining to a new fractional derivative. Appl. Math. Comput. 316, 504-515 (2018)

24. Singh, J., Kumar, D., Baleanu, D.: New aspects of fractional Biswas-Milovic model with Mittag-Leffler law. Math. Model. Nat. Phenom. 14(3), 303 (2019)

25. Singh, J.: A new analysis for fractional rumor spreading dynamical model in a social network with Mittag-Leffler law. Chaos 29, 013137 (2019)

26. Singh, J., Kumar, J., Baleanu, D.: On the analysis of fractional diabetes model with exponential law. Adv. Differ. Equ. (2018). https://doi.org/10.1186/s13662-018-1680-1

27. Das, A.K., Roy, T.K.: Fractional order generalized EPQ model. Int. J. Comput. Appl. Math. 12(2), 525-536 (2017)

28. Das, A.K., Roy, T.K.: Fractional order EOQ model with linear trend of time-dependent demand. Int. J. Intell. Syst. Appl. 3 44-53 (2015)

29. Pakhira, R., Ghosh, U., Sarkar, S.: Study of memory effects in an inventory model. Appl. Math. Sci. 12(17), 797-824 (2018)

30. Das, A.K., Roy, T.K.: Role of fractional calculus to the generalized inventory model. J. Glob. Res. Comput. Sci. 5(2), 11-23 (2014)

31. Pakhira, R., Ghosh, U., Sarkar, S.: Application of memory effects in an inventory model with linear demand and no shortage. Int. J. Res. Advent Technol. 6(8), 1853-1871 (2018)

32. Singh, J., Kumar, D., Baleanu, D., Rathore, S.: An efficient numerical algorithm for the fractional Drinfeld-Sokolov-Wilson equation. Appl. Math. Comput. 335, 12-24 (2018)

33. Iyiola, O.S., Asante-Asamani, E.O., Wade, B.A.: A real distinct poles rational approximation of generalized Mittag-Leffler functions and their inverses: applications to fractional calculus. J. Comput. Appl. Math. 330(1), 307-317 (2018)

\section{Submit your manuscript to a SpringerOpen ${ }^{\circ}$ journal and benefit from:}

- Convenient online submission

- Rigorous peer review

- Open access: articles freely available online

- High visibility within the field

- Retaining the copyright to your article

Submit your next manuscript at springeropen.com 